\title{
Exposição à violência, qualidade de vida, depressão, e burnout entre estudantes de medicina em uma universidade estadual paulista
}

\author{
Exposure to violence, quality of life, depression and burnout among medical \\ students in a state university of São Paulo, Brazil
}

\author{
Maria Fernanda Tourinho Peres ${ }^{1}$, Abraão Deyvid Lima Barreto ${ }^{2}$, Fernanda Babler ${ }^{3}$, \\ Irene Yamamoto do Vale Quaresma ${ }^{4}$, Juliana Naomy Lacerda Arakaki ${ }^{5}$, José Eluf-Neto ${ }^{6}$
}

Peres MFT, Barreto ADL, Babler F, Quaresma IYV, Arakaki JNL, Eluf-Neto J. Exposição à violência, qualidade de vida, depressão, e burnout entre estudantes de medicina em uma universidade estadual paulista / Exposure to violence, quality of life, depression and burnout among medical students in a state university of São Paulo, Brazil. Rev Med (São Paulo). 2014 jul.-set.;93(3):115-24.

RESUMO: Estudos demonstram que exposição à violência durante a formação médica tem impacto negativo na saúde mental dos estudantes. Inexistem estudos nacionais que busquem estimar a ocorrência de exposição à violência e investigar o seu impacto na saúde mental dos alunos. O nosso objetivo é descrever a exposição à violência grave por sexo e turma e investigar a associação entre exposição à violência grave e depressão, burnout e qualidade de vida dos alunos. MÉTODOS: Estudo de corte-transversal realizado em universidade pública no Estado de São Paulo. Todos os alunos do primeiro ao sexto ano $(\mathrm{n}=1072)$ receberam email com convite eletrônico e link para acesso ao questionário e 338 (31,5\%) concordaram em participar do projeto. As associações entre exposição à violência severa e depressão, burnout e qualidade de vida foram avaliadas através de modelos de regressão logística multinomial simples e ajustado por sexo e ciclo (Básico, pré-clínico e clínico). RESULTADOS: Exposição à violência constitui-se em fator de risco para depressão, burnout e má avaliação da qualidade de vida. As associações mantiveram-se significantes mesmo após ajuste para sexo e momento do curso. Exposição à violência considerada grave aumenta a chance de depressão leve em $75 \%$ e de depressão severa em 598\%. A associação com burnout aponta para um aumento na chance de burnout severo de 2,33 vezes e os alunos expostos avaliam sua qualidade de vida pior do que os alunos não expostos, sendo a chance de uma avaliação regular ou negativa entre 4 e 5 vezes maior. CONCLUSÕES: Exposição à violência durante o curso contribui para a ocorrência de depressão, burnout e má avaliação da qualidade de vida entre estudantes de medicina. Medidas que visem prevenir a ocorrência de situações abusivas e violentas e acolher e tratar as vítimas mostram-se fundamentais para melhoria das condições de saúde e qualidade de vida.

Descritores: Violência/psicologia; Estudantes de medicina/ psicologia; Depressão; Esgotamento profissional; Qualidade de vida; Saúde mental/educação.

Instituição onde o trabalho foi realizado: Faculdade de Medicina da USP. Projeto desenvolvido com financiamento da FAPESP, auxílio regular à pesquisa (processo no. 2013/11989-3).

1.Prof. Dra. do Departamento de Medicina Preventiva da Faculdade de Medicina da Universidade de São Paulo - FMUSP. E-mail: mftperes@ usp.br.

2. Acadêmico de medicina da Faculdade de Medicina da Universidade de São Paulo - FMUSP; Programa de Formação em Pesquisa na graduação do Departamento de Medicina Preventiva da FMUSP. E-mail: abraaousp100@gmail.com.

3. Acadêmica de medicina da Faculdade de Medicina da Universidade de São Paulo - FMUSP; Programa de Formação em Pesquisa na graduação do Departamento de Medicina Preventiva da FMUSP. Bolsista de Iniciação Científica da FAPESP (processo no. 2013/08795-2). E-mail: fbabler@gmail.com.

4. Acadêmica de medicina da Faculdade de Medicina da Universidade de São Paulo - FMUSP; Programa de Formação em Pesquisa na graduação do Departamento de Medicina Preventiva da FMUSP. Bolsista de Iniciação Científica PIBIC. E-mail: ireneyamamoto100@gmail. com.

5. Acadêmica de medicina da Faculdade de Medicina da Universidade de São Paulo - FMUSP; Programa de Formação em Pesquisa na graduação do Departamento de Medicina Preventiva da FMUSP. Bolsista de Iniciação Científica da FAPESP (processo no. 2013/08788-6). Email: arakaki.naomy@gmail.com.

6. Professor Titular do Departamento de Medicina Preventiva da Faculdade de Medicina da Universidade de São Paulo - FMUSP. E-mail: jelufnet@usp.br.

Endereço para correspondência: Maria Fernanda Tourinho Peres. Departamento de Medicina Preventiva - FMUSP. Av. Dr Arnaldo, $455,2^{\circ}$ andar - sala 2177. São Paulo, SP. E-mail: mftperes@usp.br. 
ABSTRACT: Studies show that exposure to violence during medical education has a negative impact on the mental health of students. There are no national studies that seek to estimate the occurrence of exposure to violence and investigate its impact on mental health of students. Our goal is to describe the exposure to severe violence by gender and class and investigate the association between exposure to severe violence and depression, burnout and quality of life of students. METHODS: Cross sectional study conducted in public university in the state of São Paulo. All students from first to sixth year $(n=1072)$ received email with electronic invitation and link to access the questionnaire and $338(31.5 \%)$ agreed to participate in the project. The associations between exposure to severe violence and depression, burnout and quality of life were evaluated by simple and adjusted for sex and cycle (Basic, pre-clinical and clinical) multinomial logistic regression models. RESULTS: Exposure to violence constitutes a risk factor for depression, burnout and poor assessment of quality of life. The associations remained

\section{INTRODUÇÃO}

A Organização Mundial de Saúde (OMS) ${ }^{1}$ define violência como "O uso intencional da força física ou do poder, real ou em ameaça, contra si mesmo, outra pessoa, ou contra um grupo ou comunidade, que resulte ou tenha possibilidade de resultar em lesão, morte, dano psicológico, mau desenvolvimento ou privação". O termo inclui atos violentos de diversas naturezas, que podem ocorrer em diferentes espaços institucionais e envolver pessoas com distintos graus de relação, desde familiares até desconhecidos. A violência interpessoal comunitária, segundo a OMS, envolve pessoas sem relação de parentesco, ocorre preferencialmente em ambientes públicos e institucionais como escolas ou outras instituições acadêmicas englobando atos de natureza física, sexual e psicológica. Neste sentido, adotamos para este artigo a definição proposta pela OMS para investigar a exposição dos estudantes à violência durante o curso médico e explorar a sua associação com a saúde mental (depressão e burnout) e a qualidade de vida dos alunos.

Em 1996 a Organização Mundial de Saúde, em sua 49a Assembleia Mundial, declarou que a violência é um problema de saúde pública de esfera global. No cerne desta afirmação está a constatação de que a exposição à violência, seja familiar ou comunitária, de natureza física, sexual ou psicológica, gera custos elevados ao setor saúde, é uma importante causa de morte na população e associa-se a problemas de saúde física e mental como depressão, ansiedades, transtornos fóbicos e transtorno de estresse pós-traumático.

No final da década de 1980 um estudo pioneiro realizado por Sheehan ${ }^{2}$ nos Estados Unidos da América demonstrou uma alta prevalência de exposição à violência (assédio, discriminação, abusos e outras formas de agressão) entre estudantes durante a formação médica. Desde então a ocorrência de situações abusivas e violentas vem sendo estudada em diversos países, com significant even after adjustment for sex and class. Exposure to severe violence increases the chance of mild depression in $75 \%$ and severe depression in 598\%. The association with burnout points to an increase in the chance of severe burnout of 2.33 times and exposed students evaluate their quality of life worse than students not exposed. The chance of a regular or negative evaluation is between 4 and 5 times greater in those exposed. CONCLUSIONS: Exposure to violence during the course contributes to the occurrence of depression, burnout and poor assessment of quality of life among medical students. Measures to prevent the occurrence of abusive and violent situations and treat victims seems fundamental to improve the health and quality of life.

Keywords: Violence/psychology; Students, medical/ psychology; Depression; Burnout, professional; Quality of life; Mental health/education.

resultados que corroboram a alta prevalência de exposição à violência durante o curso, tentando compreender os seus determinantes e as consequências para a saúde mental, a qualidade de vida e a conduta profissional do futuro médico. Os diferentes estudos demonstram que situações classificadas como assédio moral, discriminações, humilhações, perseguições, agressões físicas e sexuais conformam um conjunto de situações violentas que envolvem alunos, professores, residentes, enfermeiros e outros profissionais de saúde, além de pacientes e seus familiares.

As prevalências encontradas variam entre os diferentes estudos, o que se explica pelas diferentes metodologias adotadas. Entretanto, os diferentes estudos concordam que as prevalências são elevadas. Segundo Baldwin et al. ${ }^{3}$, em estudo realizado com uma amostra de alunos de 10 escolas médicas, $96,5 \%$ dos estudantes referiram ter sofrido ao menos um tipo de violência psicológica (assédio, discriminação ou maus-tratos) durante o curso médico. No início dos anos 2000 a prevalência mantinha-se elevada, como demonstraram Frank et al. ${ }^{4}$ em estudo realizado em 16 escolas médicas norte-americanas: $84 \%$ dos alunos referiram terem sido humilhados ou depreciados pelo menos uma vez durante o curso. Prevalências bastante elevadas foram descritas também na Nigéria $(98,5 \%)^{5}$, no Chile $(91,5 \%)^{6}$, na Alemanha $(88,5)^{7}$ e no Brasil $(92,3 \%)^{8}$. Em uma recente metanálise Fnais ${ }^{9}$ encontrou uma prevalência agrupada de humilhação e discriminação de $59,6 \%$ [95\%CI $(49,2 \% ; 68 \%)]$ e de ameaça verbal de $68,8 \%$ [95\%CI $(56,6 \% ; 80,9 \%)]$.

Estudos demostram que exposição à violência no ambiente acadêmico durante a formação médica tem um impacto negativo na auto-estima e na saúde mental dos estudantes de medicina: sentimentos de insegurança e medo, uso abusivo de álcool, sintomas depressivos, 
pensamentos suicidas, depressão, estresse, e burnout estão entre os possíveis efeitos negativos na saúde mental dos alunos $^{4-6,10-13}$. Inexistem, entretanto, estudos realizados no Brasil que busquem estimar a ocorrência de exposição à violência e investigar o seu impacto na saúde mental dos estudantes. O nosso objetivo, neste artigo, é descrever a exposição à violência grave por sexo e turma e investigar a associação entre exposição à violência grave e depressão, burnout e qualidade de vida dos alunos.

\section{METODOLOGIA}

Este estudo é parte do projeto QUARA - Qualidade das Relações no Ambiente Acadêmico e seu impacto na saúde mental e na qualidade de vida de estudantes de medicina - um estudo de corte-transversal realizado em uma universidade pública localizada no Estado de São Paulo. Todos os alunos do primeiro ao sexto ano do curso médico $(n=1072)$ receberam um email com convite eletrônico e link para acesso ao questionário e 338 (31,5\%) concordaram em participar do projeto; como 31 alunos não responderam ao questionário de maus-tratos e abusos, a amostra final foi composta por 317 alunos. O projeto foi aprovado pelo Comitê de Ética em Pesquisa da Faculdade de Medicina da Universidade de São Paulo (Ref 345.993, 31/07/2013). Acesso ao questionário foi dado àqueles que registraram a concordância em termo de consentimento informado livre e esclarecido (TCLE). Nenhuma informação pessoal foi solicitada aos alunos respondentes.

Para formulação do questionário e coleta de dados utilizamos plataforma Research Eletronic Data Capture (REDCap) $)^{14}$. O questionário on-line compreendia 12 seções temáticas: dados sociodemográficos e socioeconômicos, problemas emocionais durante a infância e adolescência; características do curso médico; exposição à violência, hábitos de vida; eventos de vida relacionados ao estresse; condição de saúde; qualidade de vida, depressão, síndrome de burnout, apoio social e conduta e atitudes profissionais.

Os dados foram coletados durante 12 semanas (11 de setembro a 23 de dezembro de 2013). Durante esse período um amplo trabalho de divulgação do projeto foi realizado através de páginas no facebook, envio de emails, distribuição de flyers e visita às salas de aula.

\section{Variáveis de estudo}

\section{Exposição à violência severa}

Para medir a exposição à violência utilizamos o questionário "Perception of Medical Students on their Learning Environment" proposto por Rautio et al. ${ }^{15}$. O questionário foi traduzido para o português por dois tradutores independentes e aborda os seguintes atos de violência por diferentes autores (professores, estudantes, residentes, preceptores / supervisores, médicos assistentes, enfermeiros, outros profissionais de saúde, pacientes ou seus familiares e outros): gritos/berros, depreciação/humilhação, atribuição de tarefas com fins punitivos, comentários depreciativos sobre a carreira, a discriminação religiosa/racial; ameaça de dano, ameaça de agressão física, assédio e discriminação sexual e violência física (tapa, chute ou bater). As respostas variam de 0 (nunca) a 4 (muitas vezes - 5 vezes ou mais). Perguntas adicionais avaliam se a violência foi considerada grave ou não pelos alunos. Com base nas respostas criamos a variável exposição à violência severa, classificada em: 0 - não foi vitima ou a violência sofrida não foi considerada severa e 1- a violência sofrida foi considerada severa ou muito severa.

\section{Depressão}

Para avaliar a presença de depressão utilizamos o Patient Health Questionnaire-9 (PHQ-9). O PHQ-9 é um questionário usado para o rastreamento de depressão que possui nove questões baseadas nos nove sintomas de depressão descritos pelo DSM-IV. As respostas são dadas pela frequência da ocorrência dos sintomas nos 15 dias anteriores à aplicação, variando de nenhuma vez (0) a quase todos os dias (3). O score final varia de 0 a 27 . Com base nos escores o grau de depressão foi classificado em 3 categorias: 0- sem sintomas depressivos (score 0 a 4), 1Sintomas depressivos ou depressão leve/moderada (escore 5 a 14), 2- Depressão grave (escore $>15$ ).

\section{Burnout}

A presença de burnout foi medida através do Maslach Burnout Inventory Students Survey (MBI-SS) ${ }^{16}$. O MBI-SS é composto por quinze questões, onde as respostas variam numa escala de sete pontos, entre "nunca" $(0)$ e "sempre" (6), e contempla assuntos como: sensação de esgotamento pelos estudos; diminuição do interesse nos estudos; e questionamento do sentido e da importância dos estudos; entre outros. Utilizamos a tradução proposta por Carloto e Câmara ${ }^{17}$, que foi adaptada para uso no Brasil.

Para se calcular a prevalência de burnout foram consideradas as suas três dimensões (esgotamento, descrença e eficácia). Inicialmente somaram-se as respostas dadas em cada uma das dimensões, obtendo-se um escore. Os casos foram classificados como leves, moderados e graves dentro de cada uma das dimensões, seguindo-se a divisão da distribuição em tercis. Foram considerados graves os casos que ao menos duas dimensões eram graves; moderados os casos com ao menos duas dimensões moderadas e leves aqueles com ao menos duas dimensões leves.

\section{Qualidade de vida}

Para avaliação das percepções sobre a qualidade de vida utilizamos o WHO-QOL-bref, questionário composto por 26 perguntas. As respostas seguem uma escala likert de 1 a 5 , onde quanto maior a pontuação, melhor a qualidade 
Peres MFT, et al. Exposição à violência, qualidade de vida, depressão, e burnout entre estudantes de medicina.

de vida. As duas questões iniciais são gerais e as outras 24 se dividem em quatro domínios da qualidade de vida (físico, psicológico, relações sociais e meio ambiente). Um escore de qualidade de vida geral foi calculado pela média das respostas e, em seguida, convertido para uma escala de 0 a 100. Para transformar em variável categórica seguimos a classificação proposta pela Organização Mundial de Saúde, assim definida: necessita melhorar (escore entre 1 e 2,9); regular (escore entre 3 e 3,9); boa (escore entre 4 e 4,9) e muito boa (escore $=5)$.

\section{Análise estatística}

A análise dos dados foi realizada no programa STATA 13.0. Inicialmente procedemos à análise descritiva, calculando medidas de frequência simples e relativa das variáveis categóricas e média e desviopadrão das variáveis quantitativas contínuas e discretas. Para análise da associação entre exposição à violência ou medidas de desfecho com sexo utilizamos o teste de chi-quadrado ou o teste exato de Fisher, quando indicado. Para análise da associação entre exposição à violência ou medidas de desfecho com ciclo (básico, pré-clinico e clínico) utilizamos o teste de Cuzik para análise de tendência ${ }^{18}$. Uma vez que utilizamos uma amostra não probabilística, cuja participação foi voluntária, os cálculos das medidas de frequência (prevalência) e associação (odds ratio) foram realizados após ajuste por ponderação pós-estratificada utilizando-se do método Raking, considerando a distribuição do total de alunos da Faculdade por sexo e turma. Como medida de precisão das estimativas pontuais foram calculados Intervalos de confiança de 95\%. As associações entre exposição à violência severa e depressão, burnout e qualidade de vida foram avaliadas através de modelos de regressão logística multinomial simples e ajustado por sexo e ciclo. A regressão logística multinomial foi selecionada por se mostrar mais adequada quando trabalhamos com desfechos categóricos não-binários.

\section{RESULTADOS}

A Tabela 1 descreve as características gerais da amostra. Dos alunos que responderam, a maioria era do sexo feminino $(50,79 \%)$. A média de idade foi 22,24 anos $( \pm 2,89)$ e $71,61 \%$ da amostra tinha menos do que 24 anos. Dos 317 alunos, cerca de 22\% receberam bônus no vestibular, a grande maioria se declarou branca $(75,08 \%)$ e pouco mais da metade está solteira $(53,31 \%)$. Os participantes do ciclo básico predominaram (44,76\%), seguidos pelos alunos do ciclo clínico $(32,81 \%)$ e, por fim, os internos $(22,40 \%)$.
Tabela 1. Características gerais da amostra $(n=317)$. São Paulo, 2013

\begin{tabular}{lcc}
\hline Características & $\mathbf{n}$ & $\mathbf{\%}$ \\
\hline Sexo & 161 & 50,79 \\
Feminino & 156 & 49,21 \\
Masculino & & \\
Idade & 227 & 71,61 \\
Até 23 anos & 90 & 28,39 \\
24 anos ou mais & & \\
Bônus no Vestibular & 248 & 78,23 \\
Não & 69 & 21,77 \\
Sim & & \\
Cor & 238 & 75,08 \\
Branca & 79 & 24,92 \\
Outra & & \\
Status de Relacionamento & 169 & 53,31 \\
Solteiro & 148 & 46,69 \\
Com companheiro/Namorado & & \\
Ciclo & 142 & 44,79 \\
Básico & 71 & 22,4 \\
Pré-clínico & & \\
Clínico & & \\
\hline & & \\
\hline
\end{tabular}

A frequência de exposição à violência severa, percepção sobre a qualidade de vida, depressão e burnout podem ser vistas na Tabela 2. Quase dois terços dos alunos $(64,25 \%)$ que responderam ao questionário foram vitimas de agressões consideradas severas. Apenas 14,69\% da amostra avaliou sua qualidade de vida como boa, enquanto o restante a considerou como regular ou necessita melhorar. A maioria dos participantes apresentou depressão leve a moderada $(56,04 \%)$, enquanto depressão severa foi encontrada em $8,27 \%$ dos alunos. Finalmente, mais da metade dos alunos apresenta algum tipo de burnout, sendo que $31,19 \%$ apresentaram burnout moderado e $25,08 \%$ severo.

Embora a proporção de alunos do sexo feminino que se declararam vitima de violência severa $(68,32 \%)$ tenha sido superior à encontrada entre alunos do sexo masculino $(58,79 \%)$, a diferença não se mostrou significante (Tabela 3). O mesmo pode ser dito sobre a distribuição de depressão entre os sexos: uma proporção maior de mulheres apresentam depressão grave $(11,25 \%) \mathrm{em}$ comparação aos alunos do sexo masculino $(7,74 \%)$, sendo que a diferença encontrada não se mostrou significante. A avaliação da qualidade de vida e a ocorrência de burnout foram semelhantes entre homens e mulheres. 
Tabela 2. Exposição à violência severa, qualidade de vida, depressão e burnout entre estudantes de medicina. São Paulo, 2013

\begin{tabular}{lcc}
\hline Variável & \% & IC95\% \\
\hline Violência severa & & \\
Não & 35,74 & $(30,33 \% ; 41,53 \%)$ \\
Sim & 64,25 & $(58,46 \% ; 69,66 \%)$ \\
Qualidade de Vida & & \\
$\quad$ Boa & 14,69 & $(10,82 \% ; 19,64 \%)$ \\
$\quad$ Regular & 62,86 & $(56,99 \% ; 68,38 \%)$ \\
$\quad$ Necessita Melhorar & 22,43 & $(18,03 \% ; 27,56 \%)$ \\
Depressão & & \\
$\quad$ Sem depressão & 36,68 & $(30,10 \% ; 41,66 \%)$ \\
$\quad$ Leve a moderada & 56,04 & $(50,08 \% ; 61,83 \%)$ \\
$\quad$ Moderadamente & 8,27 & $(5,71 \% ; 11,82 \%)$ \\
severa a severa & & \\
Burnout & 43,71 & $(37,92 \% ; 49,67 \%)$ \\
$\quad$ Sem burnout/Leve & 31,19 & $(25,98 \% ; 36,93 \%)$ \\
Moderado & 25,08 & $(20,44 \% ; 30,38 \%)$ \\
$\quad$ Severo & &
\end{tabular}

Tabela 3. Exposição à violência severa, qualidade de vida, depressão e burnout em estudantes de medicina segundo sexo. São Paulo, 2013

\begin{tabular}{lccccc}
\hline & \multicolumn{2}{c}{ Masculino } & \multicolumn{2}{c}{ Feminino } & \multirow{2}{*}{$\begin{array}{c}\text { Valor } \\
\text { de p* }\end{array}$} \\
\cline { 2 - 5 } & $\mathbf{n}$ & $\mathbf{\%}$ & $\mathbf{n}$ & $\mathbf{\%}$ & \\
\hline $\begin{array}{l}\text { Exposição à } \\
\text { violência grave }\end{array}$ & & & & & \\
Não & 64 & 41,03 & 51 & 31,68 & 0,08 \\
Sim & 92 & 58,97 & 110 & 68,32 & \\
Qualidade de vida & & & & & \\
Boa & 24 & 15,48 & 18 & 11,54 & \\
Regular & 95 & 61,29 & 98 & 62,82 & 0,57 \\
Necessita melhorar & 36 & 23,23 & 40 & 25,64 & \\
Depressão & & & & & \\
Sem depressão & 58 & 37,42 & 43 & 26,88 & \\
Leve/moderada & 85 & 54,84 & 99 & 61,88 & 0,11 \\
Grave & 12 & 7,74 & 16 & 11,25 & \\
Burnout & & & & & \\
\hline Sem burnout/leve & 66 & 42,86 & 65 & 40,88 & \\
Moderado & 44 & 28,57 & 53 & 33,33 & 0,64 \\
Severo & 44 & 28,57 & 41 & 25,79 & \\
*Qui-quadrado de pearson & & & & & \\
\hline
\end{tabular}

Na Tabela 4 observa-se a distribuição das variáveis de exposição e desfechos segundo momento do curso (ciclo básico, pré-clinico e clínico). Há uma aumento gradativo, e significante, na exposição à violência considerada severa ao longo do curso, passando de 58,5\% entre alunos do ciclo básico para $73,24 \%$ entre alunos do ciclo clínico. A prevalência de depressão grave e burnout severo, por sua vez, são mais elevadas entre alunos do ciclo básico. Alunos do ciclo básico avaliam pior a sua qualidade de vida: $30,71 \%$ consideram que a qualidade de vida necessita melhorar, comparativamente a $17,8 \%$ dos alunos no ciclo pré-clínico e $21,4 \%$ dos alunos no ciclo clínico. Esses resultados, entretanto, não se mostraram significantes.

Tabela 4. Exposição à violência severa, qualidade de vida, depressão e burnout em estudantes de medicina segundo ciclo. São Paulo, 2013

\begin{tabular}{|c|c|c|c|c|c|c|c|}
\hline & \multicolumn{2}{|c|}{ Básico } & \multicolumn{2}{|c|}{ Pré-clínico } & \multicolumn{2}{|c|}{ Clínico } & \multirow{2}{*}{$\begin{array}{l}\text { Valor } \\
\text { de p* }\end{array}$} \\
\hline & $\mathbf{n}$ & $\%$ & $\mathbf{n}$ & $\%$ & $\mathbf{n}$ & $\%$ & \\
\hline \multicolumn{8}{|c|}{$\begin{array}{l}\text { Exposição à } \\
\text { violência severa }\end{array}$} \\
\hline Não & 59 & 41,55 & 37 & 35,58 & 19 & 26,76 & \\
\hline Sim & 83 & 58,45 & 67 & 64,42 & 52 & 73,24 & 0,04 \\
\hline \multicolumn{8}{|l|}{$\begin{array}{l}\text { Qualidade } \\
\text { de vida }\end{array}$} \\
\hline Boa & 15 & 10,71 & 18 & 17,82 & 9 & 12,86 & \\
\hline Regular & 82 & 58,57 & 65 & 64,36 & 46 & 65,71 & 0,08 \\
\hline $\begin{array}{l}\text { Necessita } \\
\text { melhorar }\end{array}$ & 43 & 30,71 & 18 & 17,82 & 15 & 21,43 & \\
\hline \multicolumn{8}{|l|}{ Depressão } \\
\hline $\begin{array}{l}\text { Sem } \\
\text { depressão }\end{array}$ & 33 & 23,4 & 40 & 38,83 & 28 & 39,44 & \\
\hline $\begin{array}{l}\text { Leve/ } \\
\text { moderada }\end{array}$ & 90 & 63,83 & 55 & 53,4 & 39 & 54,93 & 0,003 \\
\hline Grave & 18 & 12,77 & 8 & 7,77 & 4 & 5,63 & \\
\hline \multicolumn{8}{|l|}{ Burnout } \\
\hline $\begin{array}{l}\text { Sem burnout/ } \\
\text { leve }\end{array}$ & 48 & 43,53 & 53 & 51,46 & 30 & 42,25 & \\
\hline Moderado & 40 & 28,78 & 30 & 29,13 & 27 & 38,03 & 0,015 \\
\hline Severo & 51 & 36,69 & 20 & 19,42 & 14 & 19,72 & \\
\hline
\end{tabular}

As associações entre exposição à violência severa e percepção sobre a qualidade de vida, depressão e burnout podem ser vistas na Tabela 5. Exposição à violência severa mostrou-se associada à uma percepção negativa sobre a qualidade de vida mesmo após ajuste para sexo e ciclo. Ser vitima de violência considerada severa aumenta 
Peres MFT, et al. Exposição à violência, qualidade de vida, depressão, e burnout entre estudantes de medicina.

chance de avaliar a qualidade de vida como regular em 4 vezes e como negativa (necessita melhorar) em quase cinco vezes (ORaj=4,97). Em ambos os casos a associação se mostrou significante e de grande magnitude, como pode ser percebido através dos Intervalos de Confiança de 95\%. Há, ainda, uma possível tendência na associação, observando-se um aumento na OR entre as categoriais de avaliação da qualidade de vida.

Exposição à violência severa também se mostrou positivamente associada à depressão, com aumento da chance de depressão leve/moderada em $75 \%$ e de depressão severa de 598\% entre expostos. Os resultados foram significantes. A associação com Burnout, embora de menor magnitude, também pode ser demonstrada. Após ajuste para sexo e turma, a associação entre exposição à violência severa e Burnout moderado perdeu significância. Entretanto, a associação com burnout severo manteve-se significante: alunos expostos apresentam uma chance 2,33 vezes maior de apresentar Burnout severo.

Tabela 5. Associação entre exposição a violência severa e qualidade de vida, depressão e Burnout entre estudantes de medicina. São Paulo, 2013

\begin{tabular}{|c|c|c|c|c|}
\hline \multirow[b]{2}{*}{ Variável desfecho } & \multicolumn{2}{|c|}{ Bruto } & \multicolumn{2}{|c|}{ Ajustado* } \\
\hline & OR & I.C. $95 \%$ & OR & I.C. $95 \%$ \\
\hline \multicolumn{5}{|l|}{ Qualidade de Vida } \\
\hline Boa & 1 & & 1 & \\
\hline Regular & 3,9 & $(1,86 ; 8,35)$ & 4,06 & $(1,87 ; 8,84)$ \\
\hline Necessita melhorar & 4,5 & $(1,93 ; 10,51)$ & 4,97 & $(2,07 ; 11,92)$ \\
\hline \multicolumn{5}{|l|}{ Depressão } \\
\hline Sem depressão & 1 & & 1 & \\
\hline Leve a moderada & 1,61 & $(0,95 ; 2,72)$ & 1,75 & $(1,01 ; 3,02)$ \\
\hline $\begin{array}{l}\text { Moderadamente severa } \\
\text { a severa }\end{array}$ & 5,85 & $(1,85 ; 18,49)$ & 6,98 & $(2,23 ; 21,82)$ \\
\hline \multicolumn{5}{|l|}{ Burnout } \\
\hline Sem burnout/Leve & 1 & & & \\
\hline Moderado & 1,81 & $(1,0 ; 3,28)$ & 1,79 & $(0,97 ; 3,3)^{* *}$ \\
\hline Severo & 2,02 & $(1,10 ; 3,71)$ & 2,33 & $(1,28 ; 4,24)$ \\
\hline
\end{tabular}

*ajustado por sexo e ciclo; ** $=0,06$

\section{DISCUSSÃO}

Os resultados obtidos neste estudo suportam a hipótese de que a exposição à violência por parte de estudantes durante a formação médica constitui-se em fator de risco para depressão, burnout e má avaliação da qualidade de vida. As associações mantiveram-se significantes mesmo após ajuste para sexo e momento do curso. Exposição à violência considerada grave pelos alunos aumenta a chance de depressão leve em $75 \%$ e de depressão severa em 598\%. A associação com burnout aponta para um amento na chance de burnout severo de 2,33 vezes e os alunos expostos avaliam sua qualidade de vida pior do que os alunos não expostos, sendo a chance de uma avaliação regular ou negativa entre 4 e 5 vezes maior entre os expostos. Encontramos ainda uma associação que se mostra maior nos casos mais graves, ou seja, as associações mostram-se mais fortes com depressão severa, 
burnout severo e pior avaliação da qualidade de vida. Os nossos resultados, portanto, apontam para a importância da exposição à violência como um dos fatores determinantes da precária saúde mental de estudantes de medicina.

O nosso estudo, entretanto, apresenta algumas limitações que devem ser levadas em consideração para uma melhor apreciação dos resultados. Inicialmente devese considerar o fato de que trabalhamos com uma amostra composta por voluntários. A opção por essa estratégia visou garantir ao máximo o anonimato dos alunos. Uma das limitações do uso de estratégias de coleta on-line através de convites e participação voluntária é a baixa taxa de resposta ${ }^{19-22}$. Gágyor et al. ${ }^{7}$, na Alemanha, obtiveram uma taxa de resposta de $32 \%$, semelhante à obtida em nosso estudo, ao realizar coleta de dados on-line para investigar a ocorrência de "experiências negativas" como abusos, maus-tratos e outras formas de agressão durante a formação médica. Além disso, estudos com voluntários sofrem, potencialmente de viés de seleção, sendo possível que os alunos que aceitaram o convite estivessem por algum motivo mobilizados a participar da pesquisa. Por isso é possível supor que haja alguma superestimação nas prevalências apresentadas. Cabe ressaltar que a taxa de resposta foi menor entre alunos do quinto e sexto anos, quando, de acordo com a literatura, a prevalência de exposição à violência tende a ser mais elevada, o que poderia, em tese, provocar uma subestimação dos resultados. Além disso, em pesquisa sobre violência ou agressões pessoas expostas tendem a não participar ou omitir violências sofridas, por medo e insegurança. Esse fenômeno também resultaria em uma subestimação dos resultados obtidos. Esse problema deve ter sido minimizado pela utilização de coleta online dos dados, através da qual a resposta poderia ser dada em ambiente externo à faculdade, facilitando o preenchimento e a declaração das situações de agressão. Soma-se a isso a ausência de questões com identificação dos estudantes, tendo sido dada total garantia de anonimato e confidencialidade aos participantes.

Encontramos, na nossa amostra, uma alta prevalência de exposição à violência: $65 \%$ dos alunos foram expostos a pelo menos uma situação de violência que consideraram grave durante o curso. $\mathrm{O}$ nosso resultado corrobora a gravidade do problema e está de acordo com o que vem demonstrando, desde a década de 1980, a literatura internacional ${ }^{2-7,10,15,23-31}$. Os nossos resultados reforçam também o achado de que estudantes de medicina apresentam altas prevalências de depressão e transtornos mentais comuns ${ }^{32-40}$, burnout ${ }^{41-43}$, e má avaliação da qualidade de vida ${ }^{44-48}$. Encontramos, em nossa amostra, uma prevalência de depressão severa de 8,27\%, 22,4\% dos alunos consideram que sua qualidade de vida está ruim e necessita melhorar e $25 \%$ apresentam burnout severo.

Mesmo considerando o possível viés de seleção e seu efeito nas estimativas de prevalências, as associações encontradas não devem ter sofrido efeito deste problema.
Isso porque nada suporta a hipótese de que nos respondentes a associação entre os fenômenos seja diferente daquela encontrada entre os não respondentes. Dessa forma, uma vez que o nosso objetivo era investigar a associação entre exposição à violência e depressão, burnout e qualidade de vida (e não estimar a prevalência desses fenômenos) consideramos pouco provável que a nossas medidas de efeito (Odds Ratio) estejam afetadas pelo possível viés.

Em nosso estudo, os alunos expostos à violência severa apresentam maior chance de avaliar que a sua qualidade de vida como regular $(\mathrm{OR}=4,06$; IC95\% $(1,87 ; 8,84))$ ou necessita melhorar $(\mathrm{OR}=4,97$; IC95\% $(2,07 ; 11,92))$, ter diagnóstico de depressão leve $(\mathrm{OR}=1,75$; IC95\% $(1,01 ; 3,02))$ ou severa $(\mathrm{OR}=6,98$; IC95\% $(2,23 ; 21,82)$, apresentar burnout severo $(\mathrm{OR}=2,33$; IC95\% $(1,28 ; 4,24))$, mesmo após ajuste para sexo e ciclo. Esse resultado reforça a hipótese de que a exposição à violência no curso médico produz efeitos negativos à saúde mental dos alunos e deve ser considerado como um dos fatores que favorecem o desenvolvimento de transtornos mentais e sofrimento psíquico entre estudantes de medicina.

Diversos estudos apontam para consequências negativas à saúde mental dos alunos decorrente da exposição à violência durante a formação médica. Alunos expostos apresentam pior auto-estima, referem mais frequentemente sintomas depressivos e ansiosos, apresentam mais frequentemente sinais de estresse e fazem uso abusivo de álcool e binge drinking ${ }^{4,5,7,10-13,49}$. Maida et al. ${ }^{6}$ encontraram associação entre problemas de saúde mental e abusos verbais, físicos, emocionais e sexuais durante a formação. Frank et al. ${ }^{4}$ observaram maior referência a estresse, humor deprimido, história clínica de depressão e ideação suicida entre os estudantes que sofreram alguma forma de depreciação durante o curso. Embora os estudos apontem para a importância da exposição à violência como um fator associado ao comprometimento da saúde mental, os resultados são apenas sugestivos, uma vez que os estudos não utilizaram instrumentos padronizados para diagnóstico dos transtornos mentais. Neste sentido, o nosso estudo representa um avanço uma vez que utilizamos instrumentos padronizados para diagnóstico de depressão, burnout e avaliação da qualidade de vida.

É importante considerar, entretanto, que os estudos são consistentes em apontar diversos fatores que, no curso médico, colaboram para a precarização da saúde mental e má avaliação da qualidade de vida. Entre os fatores de risco mais citados estão aqueles relacionados a características do curso médico como alta sobrecarga de atividades, pouco tempo para atividades de lazer, contato com doença e a morte em idade precoce, alta competitividade entre os alunos ${ }^{34,36,37,39-41,44,47}$. Há ainda estudos que ressaltam a importância de aspectos individuais dos alunos como antecedentes de problemas emocionais na infância ou adolescência, não morar com os pais ou parentes, ter um frágil apoio emocional, não possuir transporte próprio 
Peres MFT, et al. Exposição à violência, qualidade de vida, depressão, e burnout entre estudantes de medicina.

e eventos de vida relacionados ao estresse $32,35,36,38$. Para Dyrbey $^{34}$ são múltiplos os fatores que podem explicar a alta prevalência de depressão entre estudantes de medicina e informações atualmente disponíveis são insuficientes para afirmar com propriedade as causas.

Embora os nossos achados apontem para a importância da exposição à violência na determinação dos transtornos mentais e percepção negativa sobre a qualidade de vida entre estudantes de medicina, estudos adicionais mostram-se necessários, os quais devem considerar o efeito de aspectos relacionados ao curso, das características individuais dos alunos, fatores não considerados em nosso estudo. Além disso, uma vez que realizamos um estudo de corte transversal não é possível afirmar com segurança que a exposição à violência foi anterior à má avaliação da qualidade de vida ou ao desenvolvimento do quadros depressivos e de burnout. Uma forma de lidar com esta questão, em estudos de corte transversal, seria considerar a história prévia de transtornos mentais ou sofrimento psíquico seja na infância ou prévia ao ingresso no curso. Esses aspectos serão considerados em análises adicionais.

\section{CONCLUSÕES/CONSIERAÇÕES FINAIS}

Mesmo considerando as limitações acima ressaltadas, os nossos resultados reforçam a hipótese que a exposição à violência durante o curso contribui para a ocorrência de depressão, bunout e má avaliação da qualidade de vida entre estudantes de medicina. Neste sentido, medidas que visem prevenir a ocorrência de situações abusivas e violentas e acolher e tratar as vitimas mostram-se fundamentais para melhoria das condições de saúde e qualidade de vida dos alunos.

Agradecimentos: Contamos com a colaboração da Comissão de Graduação da FMUSP para coleta de dados. Agradecemos também o apoio do Centro Acadêmico Oswaldo Cruz (CAOC), do Departamento Científico (DC) e do Núcleo de Humanização do Hospital das Clínicas da Faculdade de Medicina da Universidade de São Paulo (FMUSP) para a divulgação do projeto durante a fase de coleta de dados.

\section{REFERÊNCIAS}

1. Krug EG, Dahlberg LL, Mercy JA, Zwi AB, Lozano R. World report on violence and health. Geneve: World Health Organization; 2002. p.1-18. Available from: http://www.who. int/violence_injury_prevention/violence/world_report/en/ introduction.pdf.

2. Sheehan KH, Sheehan D V, White K, Leibowitz A, Baldwin DC. A pilot study of medical student "abuse". Student perceptions of mistreatment and misconduct in medical school. JAMA. 1990;263(4):533-7. doi:10.1001/ jama.1990.03440040072031.

3. Baldwin DC, Daugherty SR, Eckenfels EJ. Student perceptions of mistreatment and harassment during medical school. A survey of ten United States schools. West J Med. 1991;155(2):140-5. Available from: http://www.ncbi.nlm.nih. gov/pmc/articles/PMC1002944/pdf/westjmed00096-0036. pdf.

4. Frank E, Carrera JS, Stratton T, Bickel J, Nora LM. Experiences of belittlement and harassment and their correlates among medical students in the United States: longitudinal survey. BMJ. 2006;333(7570):682. doi: http:// dx.doi.org/10.1136/bmj.38924.722037.7C.

5. Owoaje ET, Uchendu OC, Ige OK. Experiences of mistreatment among medical students in a university in south west Nigeria. Niger J Clin Pract. 2012;15(2):214-9. doi: 10.4103/1119-3077.97321.

6. Maida AM, Vásquez A, Herskovic V, Calderón JL, Jacard M, Pereira A, et al. A report on student abuse during medical training. Med Teach. 2003;25(5):497-501. doi: 10.1080/01421590310001606317.
7. Gágyor I, Hilbert N, Chenot JF, Marx G, Ortner T, Simmenroth-Nayda A, et al. Frequency and perceived severity of negative experiences during medical education in Germany--results of an online-survery of medical students. GMS Z Med Ausbild. 2012;29(4):Doc 55. doi: 10.3205/ zma000825.

8. Peres MFT, Deyvid A, Babler F, Quaresma IY do V, Arakaki JN, Silva ATC, et al. Violence at an academic setting and medical students' perceptions about their medical course in São Paulo, Brazil: the QUARA Project. São Paulo; 2014;17.

9. Fnais N, Soobiah C, Chen MH, Lillie E, Perrier L, Tashkhandi $M$, et al. Harassment and discrimination in medical training: a systematic review and meta-analysis. Acad Med. 2014;89(5):817-27. doi: 10.1097/ACM.0000000000000200.

10. Heru A, Gagne G, Strong D. Medical student mistreatment results in symptoms of posttraumatic stress. Acad Psychiatry. 2009;33(4):302-6. doi: 10.1176/appi.ap.33.4.302.

11. Cook AF, Arora VM, Rasinski KA, Curlin FA, Yoon JD. The prevalence of medical student mistreatment and its association with burnout. Acad Med. 2014;89(5):749-54. doi: 10.1097/ ACM.0000000000000204.

12. Haglund MEM, aan het Rot M, Cooper NS, Nestadt PS, Muller D, Southwick SM, et al. Resilience in the third year of medical school: a prospective study of the associations between stressful events occurring during clinical rotations and student well-being. Acad Med. 2009;84(2):258-68. doi: 10.1097/ACM.0b013e31819381b1.

13. Richman JA, Flaherty JA, Rospenda KM, Christensen ML. 
Mental health consequences and correlates of reported medical student abuse. JAMA. 1992;267(5):692-4. doi:10.1001/ jama.1992.03480050096032.

14. Harris PA, Taylor R, Thielke R, Payne J, Gonzalez N, Conde JG. Research electronic data capture (REDCap)-A metadatadriven methodology and workflow process for providing translational research informatics support. J Biomed Inform. 2009;42(2):377-81. doi: 10.1016/j.jbi.2008.08.010.

15. Rautio A, Sunnari V, Nuutinen M, Laitala M. Mistreatment of university students most common during medical studies. BMC Med Educ. 2005;5:36. doi: 10.1186/1472-6920-5-36.

16. Schaufeli WB, Martinez IM, Pinto AM, Salanova M, Bakker $\mathrm{AB}$. Burnout and engagement in university students: a CrossNational Study. J Cross-Cultural Psychol. 2002;33(5):464-81. doi: 10.1177/0022022102033005003.

17. Carlotto MS, Câmara SG. Características psicométricas do Maslach Burnout Inventory - Student Survey (MBISS) em estudantes universitários Brasileiros. Psico-USF. 2006;11(2):167-73. Disponível em: http://www.scielo.br/pdf/ pusf/v11n2/v11n2a05.pdf.

18. Cuzick J. A Wilcoxon-type test for trend. Stat Med. 1985;4(1):87-90.

19. Kanuk L, Berenson C. Mail Surveys and response rates: a literature review. J Mark Res. 1975;12(4):440-53. Available from: http://www.jstor.org/stable/3151093?seq=2\#page_ scan tab contents.

20. Wiersma W. The validity of surveys: online and offline. Available from: http://papers.wybowiersma.net/abstracts/ Wiersma,Wybo,The_validity_of_surveys_online_and offline.pdf.

21. Kaplowitz MD, Hadlock TD, Levine R. A comparison of web and mail survey response rates. Public Opin Q. 2004;68(1):94101. doi: $10.1093 / \mathrm{poq} / \mathrm{nfh} 006$.

22. Nulty DD. The adequacy of response rates to online and paper surveys: what can be done? Assess Eval High Educ. 2008;33(3):301-14. Available from: https://www.uaf.edu/ files/uafgov/fsadmin-nulty5-19-10.pdf.

23. Silver HK, Glicken AD. Medical student abuse. Incidence, severity, and significance. JAMA. 1990;263(4):527-32. doi: 10.1001/jama.1990.03440040066030.

24. Mangus RS, Hawkins CE, Miller MJ. Prevalence of harassment and discrimination among 1996 medical school graduates: a survey of eight US schools. JAMA. 1998;280(9):851-3.

25. Snadden D. Student health and abuse: what is going on out there? Med Teach. 2003;25(5):461-2. doi: $10.1080 / 01421590310001605615$.

26. Quince TA, Wood DF, Parker RA, Benson J. Prevalence and persistence of depression among undergraduate medical students: a longitudinal study at one UK medical school. BMJ Open. 2012;2(4):e001519. doi: 10.1136/ bmjopen-2012-001519.

27. Ahmer S, Yousafzai AW, Bhutto N, Alam S, Sarangzai AK,
Iqbal A. Bullying of medical students in Pakistan: A cross-sectional questionnaire survey. PLoS One. 2008;3(12). doi: 10.1371/journal.pone.0003889.

28. Paredes O, Sanabria-Ferrand PA, González-Quevedo LA, Moreno Realphe SP. "Bullying" en las facultades de medicina colombianas, mito o realidad. Rev Fac Med. 2010;18(2):16172. Disponível em: http://www.scielo.org.co/pdf/med/v18n2/ v18n2a03.pdf.

29. Haviland MG, Yamagata H, Werner LS, Zhang K, Dial TH, Sonne JL. Student mistreatment in medical school and planning a career in academic medicine. Teach Learn Med. 2011;23(3):231-7. doi: 10.1080/10401334.2011.586914.

30. Alzahrani HA. Bullying among medical students in a Saudi medical school. BMC Res Notes. 2012;5:335. doi: 10.1186/1756-0500-5-335.

31. Fried JM, Vermillion M, Parker NH, Uijtdehaage S. Eradicating medical student mistreatment: a longitudinal study of one institution's efforts. Acad Med. 2012;87(9):11918. doi: 10.1097/ACM.0b013e3182625408.

32. Almeida A de M, Godinho TM, Bitencourt AGV, Teles MS, Silva AS, Fonseca DC, et al. Common mental disorders among medical students. J Bras Psiquiatr. 2007;56(4):24551. http://dx.doi.org/10.1590/S0047-20852007000400002.

33. Amaral GF do, Gomide LM de P, Batista M de P, Píccolo P de P, Teles TBG, Oliveira PM de, et al. Sintomas depressivos em acadêmicos de medicina da Universidade Federal de Goiás: um estudo de prevalência. Rev Psiquiatr Rio Grande do Sul. 2008;30(2):124-30. Disponível em: http://www.scielo.br/pdf/ $\mathrm{rprs} / \mathrm{v} 30 \mathrm{n} 2 / \mathrm{v} 30 \mathrm{n} 2 \mathrm{a} 08$.

34. Dyrbye LN, Thomas MR, Shanafelt TD. Systematic review of depression, anxiety, and other indicators of psychological distress among U.S. and Canadian medical students. Acad Med. 2006;81(4):354-73. Available from: http://goo.gl/ mwRv3s.

35. Facundes VLD, Ludermir AB. Common mental disorders among health care students. Rev Bras Psiquiatr [Internet]. 2005;27(3):194-200. http://dx.doi.org/10.1590/S151644462005000300007.

36. Fiorotti KP, Rossoni RR, Borges LH, Miranda AE. Transtornos mentais comuns entre os estudantes do curso de medicina: Prevalência e fatores associados. J Bras Psiquiatr. 2010;59(1):17-23.

37. Goebert D, Thompson D, Takeshita J, Beach C, Bryson P, Ephgrave K, et al. Depressive symptoms in medical students and residents: a multischool study. Acad Med. 2009;84(2):236-41. doi: 10.1097/ACM.0b013e31819391bb.

38. Lima MCP, de Souza Domingues M, de Abreu Ramos Cerqueira AT. Prevalência e fatores de risco para transtornos mentais comuns entre estudantes de medicina. Rev Saude Publica. 2006;40(6):1035-41. http://dx.doi.org/10.1590/ S0034-89102006000700011.

39. Roberts LW. Understanding depression and distress among medical students. JAMA. 2010;304(11):1231-3. doi: 10.1001/ jama.2010.1347. 
40. Schwenk TL, Davis L, Wimsatt LA. Depression, stigma, and suicidal ideation in medical students. JAMA. 2010;304(11):1181-90. doi: 10.1001/jama.2010.1300.

41. Costa EF de O, Santos SA, Santos ATR de A, Melo EV de, Andrade TM de. Burnout Syndrome and associated factors among medical students: a cross-sectional study. Clinics (Sao Paulo). 2012;67(6):573-80. http://dx.doi.org/10.6061/ clinics/2012(06)05.

42. Prins JT, Hoekstra-Weebers JEHM, Gazendam-Donofrio SM, Dillingh GS, Bakker AB, Huisman M, et al. Burnout and engagement among resident doctors in the Netherlands: a national study. Med Educ. 2010;44(3):236-47. doi: 10.1111/j.1365-2923.2009.03590.x.

43. Santen SA, Holt DB, Kemp JD, Hemphill RR. Burnout in medical students: examining the prevalence and associated factors. South Med J. 2010;103(8):758-63. doi: 10.1097/ SMJ.0b013e3181e6d6d4.

44. Jamali A, Tofangchiha S, Jamali R, Nedjat S, Jan D, Narimani A, et al. Medical students' health-related quality of life: Roles of social and behavioural factors. Med Educ. 2013;47(10):1001-12. doi: 10.1111/medu.12247.
45. Leão PB de OES, Martins LAN, Menezes PR, Bellodi PL. Well-being and help-seeking: an exploratory study among final-year medical students. Rev Assoc Med Bras. 2011;57(4):379-86. http://dx.doi.org/10.1590/S010442302011000400009 .

46. Paro HBMS, Morales NMO, Silva CHM, Rezende CHA, Pinto RMC, Morales RR, et al. Health-related quality of life of medical students. Med Educ. 2010;44(3):227-35. doi: 10.1111/j.1365-2923.2009.03587.x.

47. Thomas MR, Dyrbye LN, Huntington JL, Lawson KL, Novotny PJ, Sloan JA, et al. How do distress and well-being relate to medical student empathy? A multicenter study. J Gen Intern Med. 2007;22(2):177-83. doi: 0.1007/s11606006-0039-6.

48. Pekmezovic T, Popovic A, Tepavcevic DK, Gazibara T, Paunic M. Factors associated with health-related quality of life among Belgrade University students. Qual Life Res. 2011;20(3):3917. doi: 10.1007/s11136-010-9754-x.

49. Mukhtar F, Daud S, Manzoor I, Amjad I, Saeed K, Naeem M, et al. Bullying of medical students. J Coll Physicians Surg Pakistan. 2010;20(12):814-8. doi: 12.2010/JCPSP.814818. 